\title{
Comet and moon solutions in the time-dependent restricted $(n+1)$-body problem
}

\author{
Carlos Barrera $\stackrel{*}{\text { Abimael Bengochea }}{ }^{\dagger}$, Carlos García-Azpeitia ${ }^{\ddagger}$
}

January 14,2020

\begin{abstract}
The time-dependent restricted $(n+1)$-body problem concerns the study of a massless body (satellite) under the influence of the gravitational field generated by $n$ primary bodies following a periodic solution of the $n$-body problem. We prove that the satellite has periodic solutions close to the large-amplitude circular orbits of the Kepler problem (comet solutions), and in the case that the primaries are in a relative equilibrium, close to small-amplitude circular orbits near a primary body (moon solutions). The comet and moon solutions are constructed with the application of a Lyapunov-Schmidt reduction to the action functional. In addition, using reversibility technics, we compute numerically the comet and moon solutions for the case of four primaries following the super-eight choreography.
\end{abstract}

\section{Introduction}

The $n$-body problem consists of $n$ masses interacting under gravitational forces. Due to the complexity of the $n$-body problem, particular cases of this problem have been thoroughly studied such as the restricted $(n+1)$-body problem, which consists of $n$ primary bodies that follow a general solution of the $n$-body problem and an extra body of negligible mass (satellite). The satellite does not influence the movement of the $n$ primaries but it is influenced by the gravitational forces of the $n$ primaries.

A simplification of the restricted $(n+1)$-body problem assumes that the $n$ primaries are in a relative equilibrium (see [1, 2, 13, 16, 20, 23]). This problem is a generalization of the classical restricted 3-body problem studied in [9, 15, 21, 22, 23]. Other works have considered the case in which the $n$ primaries follow a homographic elliptic solution [3, 6, 28, The case of the so-called Sitnikov problem considers the case of 2 primaries. While the analytic study of the restricted $(n+1)$-body problem for $n$ primaries following elliptic homographic solutions has been the focus of many research papers, few results have considered the problem where the primaries describe a general periodic solution [19].

\footnotetext{
*Depto. Matemáticas y Mecánica IIMAS, Universidad Nacional Autónoma de México, Apdo. Postal 20-726, 01000 Ciudad de México, México. crba@ciencias.unam.mx

${ }^{\dagger}$ Department of Mathematics, ITAM, Río Hondo 1, 01080, Ciudad de México, México. abimael.bengochea@itam.mx

${ }^{\ddagger}$ Depto. Matemáticas y Mecánica IIMAS, Universidad Nacional Autónoma de México, Apdo. Postal 20-726, 01000 Ciudad de México, México. cgazpe@mym.iimas.unam.mx
} 
In this work, we present an analytical study of the time-dependent restricted $(n+1)$-body problem for a general homogeneous potential, where the primary bodies describe a general periodic solution in the plane. Specifically, the Newton equation for a satellite with position $q(t) \in \mathbb{R}^{2}$ is

$$
\ddot{q}(t)=-\sum_{j=1}^{n} m_{j} \frac{q(t)-q_{j}(t)}{\left\|q(t)-q_{j}(t)\right\|^{\alpha+1}},
$$

where $q_{j}(t) \in \mathbb{R}^{2}$ represents the position of the $j$ th body with mass $m_{j}$ and $\|\cdot\|$ is the euclidean norm. We assume that $\alpha \geq 1$, where $\alpha=2$ is the gravitational case. We also consider, without loss of generality, that the solution of the $n$-body problem $q_{j}(t)$ is $2 \pi$-periodic. Two limiting problems for the satellite will be considered. In the first case, the satellite is far from the primary bodies (the comet problem). In the second case, the satellite is close to one of the primary bodies (the moon problem).

Specifically, in Theorem 11 we prove that for each integer $\mathfrak{p}$ there is an integer $\mathfrak{q}_{0}$ such that for each integer $\mathfrak{q}>\mathfrak{q}_{0}$, the comet has at least two $2 \pi \mathfrak{q}$-periodic solutions of the form

$$
q(t)=\varepsilon^{-1} e^{J(\theta+\mathfrak{p} t / \mathfrak{q})} x_{0}+\mathcal{O}(\varepsilon), \quad \varepsilon=(\mathfrak{p} / \mathfrak{q})^{2 /(\alpha+1)},
$$

where $x_{0}=(1,0) \in \mathbb{R}^{2}, J$ is the symplectic matrix, $\theta \in[0,2 \pi]$ is a phase determined by the periodic solution of the primaries and $\mathcal{O}(\varepsilon)$ is a $2 \pi \mathfrak{q}$-periodic function of order $\varepsilon$. The amplitude of the solution $\varepsilon^{-1}$ is large and the frequency $\mathfrak{p} / \mathfrak{q}$ is small. Thus the comet winds around the origin $\mathfrak{p}$ times while the primary bodies travel their orbits $\mathfrak{q}$ times.

In the moon problem we require that the primaries are in a relative equilibrium. Under this assumption we prove in Theorem 13 that for each integer $\mathfrak{q}$ there is an integer $\mathfrak{p}_{0}$ such that for each integer $\mathfrak{p}>\mathfrak{p}_{0}$, the moon has at least two $2 \pi \mathfrak{q}$-periodic solutions of the form

$$
q(t)=q_{1}(t)+\varepsilon e^{J(\theta+\mathfrak{p} t / \mathfrak{q})} x_{0}+\mathcal{O}\left(\varepsilon^{3}\right), \quad \varepsilon=(\mathfrak{p} / \mathfrak{q})^{-2 /(\alpha+1)} .
$$

The amplitude of the solution $\varepsilon$ around the first primary body is small and the frequency $\mathfrak{p} / \mathfrak{q}$ is large. Thus the moon winds around one of the primaries $\mathfrak{p}$ times while the primaries travel their periodic orbits $\mathfrak{q}$ times.

The regularized action functional of each of the problems is written (in proper coordinates) as

$$
\mathcal{A}=\mathcal{A}_{0}+\mathcal{H}: \Omega \subset H_{2 \pi}^{1}\left(\mathbb{R}^{2}\right) \rightarrow \mathbb{R}
$$

where $H_{2 \pi}^{1}$ is the Sobolev space of $2 \pi$-periodic functions. Here, $\mathcal{A}_{0}$ is the action functional of the Kepler problem and $\mathcal{H}$ is an action functional of order $\varepsilon$. The functional $\mathcal{A}_{0}$ has a $S^{1}$-set of critical points that consists of the circular orbits of the Kepler problem. The set $\Omega$ is neighborhood of the $S^{1}$-set in $H_{2 \pi}^{1}$. We prove that $\mathcal{A}$ has at least two critical points that persist from the isolated $S^{1}$-set of critical points of the functional $\mathcal{A}_{0}$ for a small parameter $\varepsilon$. This argument is based on a Lyapunov-Schmidt reduction similarly to [10, where braids of the full $N$-body problem are constructed by replacing a body in a central configuration by two bodies (see also [11]).

The gravitational case $\alpha=2$ is special because the circular orbits of the Kepler problem are not isolated due to the existence of elliptic orbits. The gravitational case is treated in Theorem 15 under the assumption that the primaries form $m$-polygons at any time. This condition is satisfied by many choreographies found in [7], for example the super-eight choreography with 

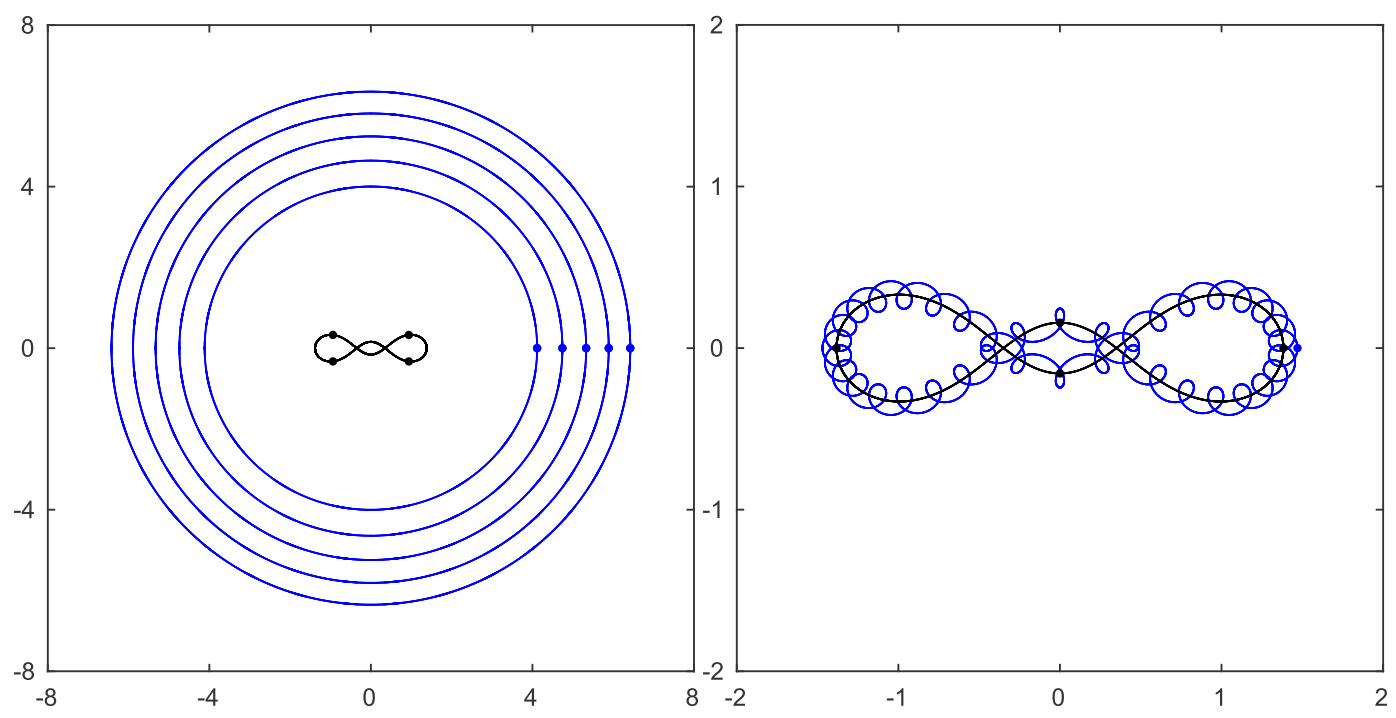

Figure 1. Left: Multiple symmetric periodic orbits of comet type in the restricted 5-body problem. Right: A symmetric periodic orbit of moon type in the restricted 5-body problem.

$n=4$ (see Remark 16), and also by many central configurations of nested polygons with a central body (see Remark 17).

The method used to prove the existence of comet and moon solutions in the time-dependent restricted body problem has limited applicability due to the assumptions: (i) In the moon case, the periodic solution of the primaries needs to be a relative equilibrium. (ii) In the gravitational case $\alpha=2$, the periodic solution of the primaries needs to be symmetric. In Section 5, we discuss briefly how to apply reversibility technics in order to show numerically the existence of comet orbits (obtained in Theorem 15) and moon orbits that cannot be obtained with our theorems. Specifically, we consider the case where four bodies with unitary mass follow the Gerver's super-eight choreography [17, 24, 26]. The fifth body is the massless particle whose dynamics is defined by the force exerted by the other four (Fig. 1). Technics of reversibility have been successfully applied for studying periodic orbits of ordinary differential equations [18; see [4, 5, 12, 25] for the case of the $N$-body problem. For more details on reversibility technics, the interested reader is referred to [19], where comet and moon orbits have been computed numerically for three primary bodies following the eight choreography.

The rest of the paper proceeds as follows. In Section 2, we set the change of variables and necessary hypothesis in order to write the functional $\mathcal{A}$ as a perturbation of the functional for the Kepler problem $\mathcal{A}_{0}$. In Section 3, we estimate the spectrum of the Hessian of $\mathcal{A}_{0}$ in Fourier components (Proposition 7), in order to make a Lyapunov-Schmidt reduction to a finite dimension. In Section 4, we prove the existence of comet and moon solutions as a consequence of our main result (Theorem 10). Theorem 10 cannot be applied directly in the gravitational case; for this reason, we obtain a separate result for the gravitational case under additional hypothesis. In Section 5, we use the reversibility technics to compute numerically the comet and moon solutions for four primaries following the super-eight choreography. 


\section{Setting up the problem}

Let $q_{j}(t) \in \mathbb{R}^{2}$ be the positions of $n$ bodies with masses $m_{j}$ for $j=1, \ldots, n$. We assume that $q_{j}(t)$ is a periodic solution of the $n$-body problem interacting under a general homogeneous potential. After rescaling space and time, we can assume, without loss of generality, that the solution $q_{j}(t)$ is $2 \pi$-periodic and that the center of mass is at the origin

$$
\sum_{j=1}^{n} m_{j} q_{j}(t)=0, \quad \sum_{j=1}^{n} m_{j} q_{j}(t)=0 .
$$

The Newton equation for a satellite is

$$
\ddot{q}(t)=-\nabla_{q} U(q, t)=-\sum_{j=1}^{n} m_{j} \frac{q(t)-q_{j}(t)}{\left\|q(t)-q_{j}(t)\right\|^{\alpha+1}},
$$

where

$$
U(q, t)=-\sum_{j=1}^{n} m_{j} \phi_{\alpha}\left(\left\|q-q_{j}\right\|\right), \quad \phi_{\alpha}(\lambda)=\frac{1}{\alpha-1} \lambda^{1-\alpha}
$$

when $\alpha>1$ and $\phi_{1}(\lambda)=-\log (\lambda)$.

We define $\mathcal{A}_{0}$ as the action for the Kepler problem in rotating coordinates,

$$
\mathcal{A}_{0}(x)=\int_{0}^{2 \pi}\left(\frac{1}{2}\left\|\left(\frac{\nu}{\omega} \partial_{\tau}+J\right) x(\tau)\right\|^{2}+\phi_{\alpha}(\|x(\tau)\|)\right) d \tau, \quad J=\left(\begin{array}{cc}
0 & 1 \\
-1 & 0
\end{array}\right) .
$$

The objective of this section is to write the action for the equation (2) as $\mathcal{A}=\mathcal{A}_{0}+\mathcal{H}$, where $\mathcal{H}$ is a small perturbation with $\mathcal{H}(x)=\mathcal{O}(\varepsilon)$. The parameter $\varepsilon^{-1}$ represents the amplitude of the comet and $\varepsilon$ the distance of the moon to a primary body.

\subsection{The comet problem}

We assume in this section, without loss of generality, that

$$
M=\sum_{j=1}^{n} m_{j}=1
$$

The Kepler problem

$$
\ddot{q}=-\frac{q}{\|q\|^{\alpha+1}}
$$

admits solutions of the form

$$
q(t)=\varepsilon^{-1} e^{J \omega t}\left(\begin{array}{l}
1 \\
0
\end{array}\right)
$$

where the frequency $\omega$ and amplitude $\varepsilon^{-1}$ satisfy the relation $\omega^{2}=\varepsilon^{(\alpha+1)}$. Note that $\varepsilon^{-1}$ is proportional to the distance between the satellite $q$ and the origin. To obtain a continuation of the large-amplitude solutions $(\varepsilon \rightarrow 0)$, we make the change of variables that scales the Kepler problem as $t=\tau / \nu$ and

$$
\begin{aligned}
q(t) & =\varepsilon^{-1} e^{J \omega \tau / \nu} x(\tau), \\
q_{j}(t) & =e^{J \omega \tau / \nu} x_{j}(\tau), \quad j=1, \cdots, n,
\end{aligned}
$$

with $\omega^{2}=\varepsilon^{\alpha+1}$. 
Proposition 1 Set $\omega^{2}=\varepsilon^{(\alpha+1)}$. For $\alpha \geq 1$, the solutions of restricted problem (2) in the coordinate $x(\tau)$ (given by (4)) are critical points of the action

$$
\mathcal{A}(x)=\mathcal{A}_{0}(x)+\mathcal{H}(x)
$$

where $\mathcal{H}(x)=\int_{0}^{2 \pi} h(x(\tau), \tau) d \tau$ with

$$
h(x, \tau)=\sum_{j=1}^{n} m_{j}\left[\phi_{\alpha}\left(\left\|x(\tau)-\varepsilon x_{j}(\tau)\right\|\right)-\phi_{\alpha}(\|x(\tau)\|)\right] .
$$

and $x_{j}(\tau)$ are given by $(5)$.

Proof. Using the change of variables (4) and $t=\tau / \nu$, the first term of (2) becomes

$$
\ddot{q}(t)=\varepsilon^{-1} \omega^{2} e^{J \omega \tau / \nu}\left(\frac{\nu}{\omega} \partial_{\tau}+J\right)^{2} x(\tau) .
$$

Replacing this term in (2) and using (5), the equation of motion becomes

$$
\left(\frac{\nu}{\omega} \partial_{\tau}+J\right)^{2} x(\tau)=-\sum_{j=1}^{n} m_{j} \frac{x(\tau)-\varepsilon x_{j}(\tau)}{\left\|x(\tau)-\varepsilon x_{j}(\tau)\right\|^{\alpha+1}} .
$$

On the other hand, if $\mathcal{A}(x)=\int_{0}^{2 \pi} A\left(\tau, x(\tau), x^{\prime}(\tau)\right) d \tau$, then

$$
\begin{aligned}
\frac{d}{d \tau} \frac{\partial A}{\partial x^{\prime}} & =\frac{\nu^{2}}{\omega^{2}} x^{\prime \prime}(\tau)+\frac{\nu}{\omega} J x^{\prime}(\tau), \\
\frac{\partial A}{\partial x} & =-\frac{\nu}{\omega} J x^{\prime}(\tau)+x(\tau)-\sum_{j=1}^{n} m_{j} \frac{x(\tau)-\varepsilon x_{j}(\tau)}{\left\|x(\tau)-\varepsilon x_{j}(\tau)\right\|^{\alpha+1}} .
\end{aligned}
$$

Using the Euler-Lagrange equation, we get equation (6).

We need to define $h(x, \tau)$ in the space of $2 \pi$-periodic functions.

\section{Proposition 2 If}

$$
\omega=\mathfrak{p} / \mathfrak{q}, \quad \nu=1 / \mathfrak{q},
$$

then the function $h(t, x)$ defined in Proposition 1 is $2 \pi$-periodic and $\nabla_{x} h(x, \tau)=\mathcal{O}\left(\varepsilon^{2}\right)$.

Proof. Since $q_{j}(t)$ is $2 \pi$-periodic, then $q_{j}(\tau / \nu)$ is $2 \pi / \mathfrak{q}$-periodic and $e^{-J \omega t / \nu}$ is a $2 \pi / \mathfrak{p}$-periodic matrix. Therefore, $x_{j}(\tau)$ is $2 \pi$-periodic and $h(x, \tau)=h(x, \tau+2 \pi)$. Since $\sum_{j=1}^{n} m_{j} x_{j}=0$, using complex identification of $x \in \mathbb{C}$, then

$$
\begin{aligned}
\nabla_{x} h(x, \tau) & =\sum_{j=1}^{n} m_{j}\left(\alpha \frac{x}{|x|^{\alpha+2}} x_{j} \varepsilon+\frac{\alpha(\alpha+1)}{2} \frac{x}{|x|^{\alpha+3}} x_{j}^{2} \varepsilon^{2}+\mathcal{O}\left(\varepsilon^{3}\right)\right) \\
& =\left(\sum_{j=1}^{n} m_{j} \frac{\alpha(\alpha+1)}{2} \frac{x}{|x|^{\alpha+3}} x_{j}^{2}\right) \varepsilon^{2}+\mathcal{O}\left(\varepsilon^{3}\right) .
\end{aligned}
$$

We look for comet solutions in which the amplitude is very large. That is, we need to take $\varepsilon \rightarrow 0$ and $\omega^{2}=\varepsilon^{(\alpha+1)} \rightarrow 0$. Because the equation of motion for $x(\tau)$ has the term $\left(\frac{\nu}{\omega} \partial_{\tau}+J\right)^{2} x(\tau)$ and $\omega \rightarrow 0$, we need $\nu / \omega \geq \delta>0$. Since $\nu / \omega=1 / \mathfrak{p}$, we achieve this by fixing $\mathfrak{p}$ and letting $\mathfrak{q} \rightarrow \infty$. 


\subsection{The moon problem}

We assume that the satellite follows the first body. After rescaling space and time we can assume, without loss of generality, that $m_{1}=1$. To find the moon solutions, we define the time scale $t=\tau / \nu$ and the change of variables

$$
\begin{aligned}
q(t) & =q_{1}(t)+\varepsilon e^{J \omega \tau / \nu} x(\tau), \\
q_{j}(t) & =e^{J \omega \tau / \nu} x_{j}(\tau), \quad j=1, \cdots, n,
\end{aligned}
$$

where $\omega^{2}=\varepsilon^{-(\alpha+1)}$.

Proposition 3 Let $\alpha \geq 1$ and $\omega^{2}=\varepsilon^{-(\alpha+1)}$. The solutions of restricted problem (2) in the coordinate $x(\tau)$ (given by (7)) are critical points of the action

$$
\mathcal{A}(x)=\mathcal{A}_{0}(x)+\mathcal{H}(x)
$$

where $\mathcal{H}(x)=\int_{0}^{2 \pi} h(x, \tau) d \tau$ with

$h(x, \tau)=\left\{\begin{array}{c}\varepsilon^{\alpha-1} \sum_{j=2}^{n} m_{j}\left[\phi_{\alpha}\left(\left\|x_{1}(\tau)-x_{j}(\tau)+\varepsilon x(\tau)\right\|\right)-\nabla \phi_{\alpha}\left(\left\|x_{1}(\tau)-x_{j}(\tau)\right\|\right) \cdot \varepsilon x(\tau)\right], \alpha>1 \\ \sum_{j=2}^{n} m_{j}\left[-\log \left(\frac{\left\|x_{1}(\tau)-x_{j}(\tau)+\varepsilon x(\tau)\right\|}{\left\|x_{1}(\tau)-x_{j}(\tau)\right\|}\right)+\frac{x_{1}(\tau)-x_{j}(\tau)}{\left\|x_{1}(\tau)-x_{j}(\tau)\right\|^{2}} \cdot \varepsilon x(\tau)\right], \alpha=1\end{array}\right.$,

and $x_{j}(\tau)$ are given by $(8)$.

Proof. Using the change of variables (7), the left side of the equation (2) becomes

$$
\ddot{q}(t)=\ddot{q}_{1}(t)+\varepsilon \omega^{2} e^{J \omega \tau / \nu}\left(\frac{\nu}{\omega} \partial_{\tau}+J\right)^{2} x(\tau) .
$$

But $q_{1}(t)$ is a solution of the $n$-body problem, therefore

$$
\ddot{q}_{1}(t)=-\sum_{j=2}^{n} m_{j} \frac{q_{1}(t)-q_{j}(t)}{\left\|q_{1}(t)-q_{j}(t)\right\|^{\alpha+1}} .
$$

Assuming $\alpha>1$ and expanding the sum in the right side of (2),

$$
\sum_{j=1}^{n} m_{j} \frac{q(t)-q_{j}(t)}{\left\|q(t)-q_{j}(t)\right\|^{\alpha+1}}=\frac{e^{J \omega \tau / \nu} x(\tau)}{\varepsilon^{\alpha}\|x(\tau)\|^{\alpha+1}}+\sum_{j=2}^{n} m_{j} \frac{q(t)-q_{j}(t)}{\left\|q(t)-q_{j}(t)\right\|^{\alpha+1}} .
$$

Replacing this in (2) and using (8), the equation of motion is

$$
\begin{aligned}
\left(\frac{\nu}{\omega} \partial_{\tau}+J\right)^{2} x(\tau) & =-\frac{x(\tau)}{\|x(\tau)\|^{\alpha+1}} \\
& -\varepsilon^{\alpha} \sum_{j=2}^{n} m_{j}\left(\frac{x_{1}(\tau)-x_{j}(\tau)+\varepsilon x(\tau)}{\left\|x_{1}(\tau)-x_{j}(\tau)+\varepsilon x(\tau)\right\|^{\alpha+1}}-\frac{x_{1}(\tau)-x_{j}(\tau)}{\left\|x_{1}(\tau)-x_{j}(\tau)\right\|^{\alpha+1}}\right) .
\end{aligned}
$$


On the other hand, if $\mathcal{A}(x)=\int_{0}^{2 \pi} A\left(\tau, x(\tau), x^{\prime}(\tau)\right) d \tau$, then

$$
\begin{aligned}
\frac{d}{d \tau} \frac{\partial A}{\partial x^{\prime}}= & \frac{\nu^{2}}{\omega^{2}} x^{\prime \prime}(\tau)+\frac{\nu}{\omega} J x^{\prime}(\tau) \\
\frac{\partial A}{\partial x}= & -\frac{\nu}{\omega} J x^{\prime}(\tau)+x(\tau)+\frac{x(\tau)}{\|x(\tau)\|^{\alpha+1}} \\
& -\varepsilon^{\alpha} \sum_{j=1}^{n} m_{j} \frac{x_{1}(\tau)-x_{j}(\tau)-\varepsilon x(\tau)}{\left\|x_{1}(\tau)-x_{j}(\tau)-\varepsilon x(\tau)\right\|^{\alpha+1}}-\frac{x_{1}(\tau)-x_{j}(\tau)}{\left\|x(\tau)-\varepsilon x_{j}(\tau)\right\|^{\alpha+1}} .
\end{aligned}
$$

Using the Euler-Lagrange equation, we get equation (9). The case $\alpha=1$ is similar.

We look for moon solutions in which the amplitude $\varepsilon$ is very small. At this point, we notice that the problem of the moon is different from the problem of the comet because $\omega \rightarrow \infty$ if $\varepsilon \rightarrow 0$. But we need $\nu / \omega>\delta>0$, then $\nu \rightarrow \infty$ as $\varepsilon \rightarrow 0$. But on the other hand, the period of $x_{j}(\tau)$ goes to infinity and $h(x, \tau)$ is not $2 \pi$-periodic. Therefore, we cannot define $\mathcal{A}$ in the space of $2 \pi$-periodic paths. In order to avoid this problem, we can consider the simpler case where the periodic solution of the primaries is a relative equilibrium. That means that $q_{j}(t)=e^{t J} a_{j}$, where

$$
a_{j}=\sum_{k=1(k \neq j)}^{n} m_{k} \frac{\left(a_{j}-a_{k}\right)}{\left\|a_{j}-a_{k}\right\|^{\alpha+1}}, \quad m_{1}=1, \quad a_{j} \in \mathbb{R}^{2} .
$$

In this case, we can choose values of $\omega$ and $\nu$ such that $h(x, \tau)$ is $2 \pi$-periodic.

\section{Proposition 4 If}

$$
\frac{\omega-1}{\nu}=\mathfrak{p} \in \mathbb{Z},
$$

and $q_{j}(t)=e^{t J} a_{j}$ with 10 , then $h(x, \tau)$ defined in the Proposition 3 is $2 \pi$-periodic. Moreover, we have and $\nabla_{x} h(x, \tau)=\mathcal{O}\left(\varepsilon^{\alpha+1}\right)$ and $\nu / \omega=\left(1-\varepsilon^{\frac{\alpha+1}{2}}\right) / \mathfrak{p}$.

Proof. If $q_{j}(t)=e^{t J} a_{j}$ and the configuration $a_{j}$ satisfies $[10$, then

$$
x_{j}(\tau)=e^{-J(\omega-1) \tau / \nu} a_{j} .
$$

Therefore $x_{j}(\tau)$ and $h(x, \tau)$ are $2 \pi$-periodic. Furthermore, from the definition of $h$ we have $\nabla h(x, \tau)=\mathcal{O}\left(\varepsilon^{\alpha+1}\right)$.

\section{Lyapunov-Schmidt reduction}

The Euler-Lagrange equations of $\mathcal{A}_{0}$ is

$$
\frac{\delta \mathcal{A}_{0}}{\delta x}(x ; \varepsilon)=\left(\frac{\nu}{\omega} \partial_{\tau}+J\right)^{2} x+\frac{x}{\|x\|^{\alpha+1}} .
$$

This equation has the circle of solutions $e^{J \theta} x_{0}$ for $\theta_{0} \in[0,2 \pi]$, where $x_{0}=(1,0)^{T}$,

$$
S^{1}=\left\{e^{J \theta} x_{0} \in H_{2 \pi}^{1}\left(\mathbb{R}^{2}\right): \theta \in[0,2 \pi]\right\} .
$$


The question that we would like to answer is if the critical solutions of $\mathcal{A}(x ; 0)=\mathcal{A}_{0}(x ; 0)$ persist for $\varepsilon \neq 0$. For this purpose we define a neighborhood $\Omega_{\rho}$ of $S^{1}$ where the functional $\mathcal{A}$ is well defined,

$$
\Omega_{\rho}:=\left\{x \in H_{2 \pi}^{1}\left(\mathbb{R}^{2}\right):\left\|x-e^{J \theta} x_{0}\right\|_{H^{1}}<\rho, \quad \theta \in[0,2 \pi]\right\}
$$

Thus our two problems are set as critical points of the action functional $\mathcal{A}_{0}$ perturbed by $\mathcal{H}$,

$$
\mathcal{A}(x ; \varepsilon)=\mathcal{A}_{0}(x ; \varepsilon)+\mathcal{H}(x ; \varepsilon): \Omega_{\rho} \times \mathbb{R} \rightarrow \mathbb{R} .
$$

Proposition 5 Assume that $\alpha \geq 1$, and $\omega$ and $\nu$ satisfy the conditions of Propositions 2 and 4. Thus the action functional (12) that gives solutions to the restricted body problem is well-defined on $\Omega_{\rho}$ for $\rho$ small enough.

Proof. It is necessary only to see that $\mathcal{A}$ is well-defined in $\Omega_{\rho}$ and that critical solutions in $\Omega_{\rho}$ are critical solutions in $H_{2 \pi}^{1}$. Since we look for critical points of the action $\mathcal{A}(x ; \varepsilon)$ in the space $H_{2 \pi}^{1}$, then $\mathcal{A}$ is well-defined in $H_{2 \pi}^{1}$ only if $\omega$ and $\nu$ satisfies the conditions of Propositions 2 and 4.

Since $H_{2 \pi}^{1} \subset C_{2 \pi}^{0}$, there is a constant $C$ such that $\|\cdot\|_{C^{0}} \leq C\|\cdot\|_{H^{1}}$. If $x \in \Omega_{\rho}$, then

$$
\max _{t}\left|x(t)-e^{J \theta} x_{0}\right|=\left\|x-e^{J \theta} x_{0}\right\|_{C^{0}} \leq C\left\|x-e^{J \theta} x_{0}\right\|_{H^{1}}<C \rho .
$$

Therefore, we choose $\rho$ small enough such that the path of $x$ belongs to an annulus with center at the origin. The nonlinear integrals of $\mathcal{A}(x)$ are bounded in the region where $h(x, t)$ is analytic, which holds in the neighborhood $\Omega_{\rho}$ because it excludes collisions by construction.

\subsection{Non-degeneracy condition}

In the moon and comet problems the functions $\nu=\nu(\varepsilon)$ and $\omega=\omega(\varepsilon)$ depend on $\varepsilon$ and satisfy

$$
\lim _{\varepsilon \rightarrow 0} \frac{\nu}{\omega}=\frac{1}{\mathfrak{p}}
$$

Since $\mathcal{H}=\mathcal{O}(\varepsilon)$, the action at $\varepsilon=0$ is

$$
\mathcal{A}(x ; 0)=\mathcal{A}_{0}(x)=\int_{0}^{2 \pi} \frac{1}{2}\left\|\left(\frac{1}{\mathfrak{p}} \partial_{\tau}+J\right) x(\tau)\right\|^{2}+\phi_{\alpha}(\|x(\tau)\|) d \tau .
$$

In this section we make necessary estimates on the Hessian $\nabla^{2} \mathcal{A}_{0}(x ; 0)$ in order to perform a Lyapunov-Schmidt reduction.

For $x \in H_{2 \pi}^{1}$, the Fourier components of $x$ are

$$
x(t)=\sum_{l \in \mathbb{Z}} \hat{x}_{l} e^{i l \tau}, \quad \hat{x}_{l}=\overline{\hat{x}}_{-l} \in \mathbb{R}^{2} .
$$

We define the components of $x$ as

$$
\xi=\hat{x}_{0}=\int_{0}^{2 \pi} x(\tau) d \tau \in X_{0}, \quad \eta=\sum_{l \neq 0} \hat{x}_{l} e^{i l \tau}=x(\tau)-\int_{0}^{2 \pi} x(\tau) d \tau \in X .
$$


If we decompose $\xi$ in polar coordinates, $\xi=r(\cos \theta, \sin \theta)$, we can write $x \in H_{2 \pi}^{1}$ as a function of the variables $(\theta, r, \eta)$, given by

$$
x(\theta, r, \eta)=r\left(\begin{array}{c}
\cos \theta \\
\sin \theta
\end{array}\right)+\eta, \quad(\theta, r, \eta) \in[0,2 \pi] \times \mathbb{R}^{+} \times X .
$$

In the new coordinates $(\theta, r, \eta)$ the action is $\mathcal{A}_{0}(\theta, r, \eta)=\mathcal{A}(\theta, r, \eta ; 0)$. In these coordinates the elements in the critical circle $S^{1}$ of $\mathcal{A}_{0}$ are

$$
x(\theta, 1,0)=e^{J \theta} x_{0} .
$$

Lemma 6 For $\alpha \geq 1$ the action functional for the Kepler problem has the expansion

$$
\mathcal{A}_{0}(\theta, r+1, \eta)=\frac{1}{2} \int_{0}^{2 \pi}(\alpha+1) r^{2}+\left\|\left(\frac{1}{\mathfrak{p}} \partial_{\tau}+J\right) \eta\right\|^{2}-\|\eta\|^{2}+(\alpha+1)\left(\left(\begin{array}{c}
\cos \theta \\
\sin \theta
\end{array}\right) \cdot \eta\right)^{2} d \tau+\mathcal{O}\left(|(\theta, r, \eta)|^{3}\right) .
$$

Proof. For $\alpha \neq 1$ the functional action for the Kepler problem is

$$
\mathcal{A}_{0}(x)=\int_{0}^{2 \pi} \frac{1}{2}\left\|\left(\frac{1}{\mathfrak{p}} \partial_{\tau}+J\right) x(\tau)\right\|^{2}+\frac{1}{\alpha-1}\|x(\tau)\|^{1-\alpha} d \tau .
$$

In order to compute the Hessian of the functional $\mathcal{A}$ around the critical solution, in the coordinates $(\theta, r, \eta)$, we expand in Taylor series the functional

$$
\mathcal{A}_{0}(\theta, r+1, \eta)=\int_{0}^{2 \pi} \frac{1}{2}(r+1)^{2}+\frac{1}{2}\left\|\left(\frac{1}{\mathfrak{p}} \partial_{\tau}+J\right) \eta\right\|^{2}+\frac{1}{\alpha-1}\left(\left\|(r+1)\left(\begin{array}{c}
\cos \theta \\
\sin \theta
\end{array}\right)+\eta\right\|^{2}\right)^{\frac{1-\alpha}{2}} d \tau
$$

We notice that

$$
\left(\left\|(r+1)\left(\begin{array}{c}
\cos \theta \\
\sin \theta
\end{array}\right)+\eta\right\|^{2}\right)^{\frac{1-\alpha}{2}}=\left(1+2 r+r^{2}+2(r+1)\left(\begin{array}{c}
\cos \theta \\
\sin \theta
\end{array}\right) \cdot \eta+\|\eta\|^{2}\right)^{\frac{1-\alpha}{2}} .
$$

Using that

$$
(1+\rho)^{\frac{1-\alpha}{2}}=1-\frac{\alpha-1}{2} \rho+\frac{\alpha^{2}-1}{8} \rho^{2}+\mathcal{O}\left(\rho^{3}\right)
$$

we get the expansion

$$
\begin{aligned}
\left(\left\|(r+1)\left(\begin{array}{c}
\cos \theta \\
\sin \theta
\end{array}\right)+\eta\right\|^{2}\right)^{\frac{1-\alpha}{2}}= & -\frac{\alpha-1}{2}\left[2 r+r^{2}+2(r+1)\left(\begin{array}{c}
\cos \theta \\
\sin \theta
\end{array}\right) \cdot \eta+\|\eta\|^{2}\right] \\
& +\frac{\alpha^{2}-1}{8}\left[4 r^{2}+4\left(\left(\begin{array}{c}
\cos \theta \\
\sin \theta
\end{array}\right) \cdot \eta\right)^{2}\right]+\mathcal{O}\left(|(\theta, r, \eta)|^{3}\right) .
\end{aligned}
$$

Since $\int_{0}^{2 \pi} \eta d \tau=0$, we obtain 13 .

For $\alpha=1$ the functional action is

$$
\mathcal{A}_{0}(x)=\int_{0}^{2 \pi} \frac{1}{2}\left\|\left(\frac{1}{\mathfrak{p}} \partial_{\tau}+J\right) x(\tau)\right\|^{2}-\log (\|x(\tau)\|) d \tau .
$$


Therefore

$$
\begin{aligned}
\mathcal{A}_{0}(\theta, r+1, \eta)=\frac{1}{2} & \int_{0}^{2 \pi}(r+1)^{2}+\left\|\left(\frac{1}{\mathfrak{p}} \partial_{\tau}+J\right) \eta\right\|^{2} \\
& -\log \left(1+2 r+r^{2}+2(r+1)\left(\begin{array}{c}
\cos \theta \\
\sin \theta
\end{array}\right) \cdot \eta+\|\eta\|^{2}\right) d \tau
\end{aligned}
$$

Using that

$$
\log (1+\rho)=\rho-\frac{1}{2} \rho^{2}+\mathcal{O}\left(\rho^{3}\right)
$$

we get the expansion

$$
\begin{aligned}
\log \left(1+2 r+r^{2}+2(r+1)\left(\begin{array}{c}
\cos \theta \\
\sin \theta
\end{array}\right) \cdot \eta+\|\eta\|^{2}\right)= & r+\frac{1}{2} r^{2}+(r+1)\left(\begin{array}{c}
\cos \theta \\
\sin \theta
\end{array}\right) \cdot \eta+\frac{1}{2}\|\eta\|^{2} \\
& -r^{2}-\left(\left(\begin{array}{c}
\cos \theta \\
\sin \theta
\end{array}\right) \cdot \eta\right)^{2}+\mathcal{O}\left(|(\theta, r, \eta)|^{3}\right)
\end{aligned}
$$

Using that $\int_{0}^{2 \pi} \eta d \tau=0$, we obtain 13 with $\alpha=1$.

Proposition 7 Assume that $\alpha \geq 1(\alpha \neq 2)$, then the Hessian of the action functional $\mathcal{A}_{0}(\theta, r, \eta)$ at $(\theta, 1,0)$ is

$$
\nabla^{2} \mathcal{A}_{0}(\theta, 1,0)=\left(\begin{array}{cc}
\nabla_{\theta}^{2} \mathcal{A}_{0} & 0 \\
0 & \nabla_{(r, \eta)}^{2} \mathcal{A}_{0}
\end{array}\right)
$$

where

$$
\nabla_{\theta}^{2} \mathcal{A}_{0}(\theta, 1,0)=0, \quad\left\|\left(\nabla_{(r, \eta)}^{2} \mathcal{A}_{0}(\theta, 1,0)\right)^{-1}\right\|_{H_{2 \pi}^{1} \rightarrow H_{2 \pi}^{1}} \leq C,
$$

and $C$ is a positive constant.

Proof. By a direct computation from (13), the Hessian is block diagonal

$$
\nabla^{2} \mathcal{A}_{0}(\theta, 1,0)=\nabla_{\theta}^{2} \mathcal{A}_{0} \oplus \nabla_{r}^{2} \mathcal{A}_{0} \oplus \nabla_{\eta}^{2} \mathcal{A}_{0},
$$

where $\nabla_{\theta}^{2} \mathcal{A}_{0}=0, \nabla_{r}^{2} \mathcal{A}_{0}=\alpha+1$ and

$$
\nabla_{\eta}^{2} \mathcal{A}_{0}=\left(-\partial_{\tau}^{2}+1\right)^{-1}\left[-\left(\frac{1}{\mathfrak{p}} \partial_{\tau}+J\right)^{2}-I+(\alpha+1)\left(\begin{array}{cc}
\cos ^{2} \theta & \cos \theta \sin \theta \\
\cos \theta \sin \theta & \sin ^{2} \theta
\end{array}\right)\right]
$$

Since $\partial_{\tau} e^{i l \tau}=i l e^{i l \tau}$, the Hessian $\nabla_{\eta}^{2} \mathcal{A}$ in Fourier components is

$$
\nabla_{\eta}^{2} \mathcal{A}_{0}(\theta, 1,0) x=\sum_{l \in \mathbb{Z} \backslash\{0\}} A_{l} \hat{x}_{l} e^{i l \tau}
$$

where

$$
A_{l}=\left(l^{2}+1\right)^{-1}\left(\begin{array}{cc}
(l / \mathfrak{p})^{2}+(\alpha+1) \cos ^{2} \theta & -2 i(l / \mathfrak{p})+(\alpha+1) \sin \theta \cos \theta \\
2 i(l / \mathfrak{p})+(\alpha+1) \sin \theta \cos \theta & (l / \mathfrak{p})^{2}+(\alpha+1) \sin ^{2} \theta
\end{array}\right) .
$$


The matrix $A_{l}$ has eigenvalues

$$
\lambda_{l}^{ \pm}=\left(l^{2}+1\right)^{-1}\left(\frac{1}{2}(\alpha+1)+(l / \mathfrak{p})^{2} \pm \frac{1}{2} \sqrt{(\alpha+1)^{2}+16(l / \mathfrak{p})^{2}}\right)
$$

The eigenvalues satisfy $\lambda_{l}^{ \pm} \neq 0$ if $l / \mathfrak{p} \neq \sqrt{3-\alpha}, 0$. This condition holds because $\alpha \neq 2$ and $l \neq 0$ for the coordinate $\eta$. Since $\lim _{l \rightarrow \infty} \lambda_{l}^{ \pm}=(1 / \mathfrak{p})^{2}$, there is a small positive constant $C_{0}$ such that $\left|\lambda_{l}^{ \pm}\right| \geq C_{0}^{-1}$ for all $l \neq 0$, i.e. $\left|A_{l}^{-1}\right| \leq C_{0}$ for $l \neq 0$. Therefore, the estimate for $\nabla_{\eta}^{2} \mathcal{A}_{0}(\theta, 1,0)$ follows from the fact that

$$
\left\|\left(\nabla_{\eta}^{2} \mathcal{A}_{0}(\theta, 1,0)\right)^{-1} x\right\|_{H_{2 \pi}^{1}}^{2}=\sum_{l \in \mathbb{Z} \backslash\{0\}}\left(l^{2}+1\right)\left|A_{l}^{-1} \hat{x}_{l}\right|^{2} \leq C_{0}^{2} \sum_{l \in \mathbb{Z} \backslash\{0\}}\left(l^{2}+1\right)\left|\hat{x}_{l}\right|^{2}=C_{0}^{2}\|x\|_{H_{2 \pi}^{1}}^{2} .
$$

Thus, the inverse of $\nabla_{(r, \eta)}^{2} \mathcal{A}_{0}(\theta, 1,0)$ is bounded by

$$
C=\max \left\{C_{0}, \frac{1}{\alpha+1}\right\} .
$$

Remark 8 In the gravitational case $\alpha=2$, the condition in the proposition does not hold because $A_{l}$ is not invertible for $l=\mathfrak{p}$. This fact is a consequence of the existence of the elliptic orbits of the Kepler problem, i.e. the circular orbits are not isolated.

\subsection{Lyapunov-Schmidt reduction}

In this section we make a Lyapunov-Schmidt reduction to finite dimension for the operator

$$
\nabla \mathcal{A}(x ; \varepsilon): \Omega_{\rho} \times \Lambda_{\varepsilon} \subset H_{2 \pi}^{1} \times \mathbb{R} \rightarrow H_{2 \pi}^{1},
$$

given by

$$
\nabla \mathcal{A}(x ; \varepsilon)=\nabla \mathcal{A}_{0}(x ; \varepsilon)+\nabla \mathcal{H}(x ; \varepsilon), \quad \nabla \mathcal{H}(x ; \varepsilon)=\mathcal{O}_{H_{2 \pi}^{1}}\left(\varepsilon^{2}\right)
$$

Since

$$
\nabla \mathcal{A}(\theta, r, \eta ; \varepsilon)=\left(\nabla_{\theta} \mathcal{A}, \nabla_{(r, \eta)} \mathcal{A}\right),
$$

the equation $\nabla \mathcal{A}=0$ is equivalent to solve $\nabla_{\theta} \mathcal{A}=0$ and $\nabla_{(r, \eta)} \mathcal{A}=0$. The Lyapunov-Schmidt reduction consist of solving from the equation $\nabla_{(r, \eta)} \mathcal{A}=0$ defined in the set $\Omega_{\rho} \times \Lambda_{\varepsilon_{0}}$, the components $(r, \eta)$ as function of $(\theta ; \varepsilon)$, where

$$
\Lambda_{\varepsilon_{0}}=\left\{\varepsilon \in \mathbb{R}: 0 \leq \varepsilon<\varepsilon_{0}\right\}
$$

Proposition 9 There is a $\rho$ and $\varepsilon_{0}$ such that $\mathcal{A}(\theta, r, \eta ; \varepsilon)$ has a critical point in $\Omega_{\rho} \times \Lambda_{\varepsilon_{0}}$ if and only if

$$
\Psi(\theta ; \varepsilon)=\mathcal{A}(\theta, r(\theta ; \varepsilon), \eta(\theta ; \varepsilon) ; \varepsilon): S^{1} \times \Lambda_{\varepsilon_{0}} \rightarrow \mathbb{R}
$$

has a critical point in $S^{1} \times \Lambda_{\varepsilon_{0}}$, where $r=r(\theta ; \varepsilon)$ and $\eta=\eta(\theta ; \varepsilon)$ is the unique solution of

$$
\nabla_{(r, \eta)} \mathcal{A}(\theta, r, \eta ; \varepsilon)=0
$$


Proof. We have that $\nabla \mathcal{A}(\vartheta, 1,0 ; 0)=0$ for any $\vartheta \in S^{1}$. By Proposition 7 , the operator $\nabla_{(r, \eta)}^{2} \mathcal{A}(\vartheta, 1,0 ; 0)$ is invertible. Then, by the Implicit Function Theorem, we can solve $r_{\vartheta}=$ $r_{\vartheta}(\theta ; \varepsilon)$ and $\eta_{\vartheta}=\eta_{\vartheta}(\theta ; \varepsilon)$ as the unique solution of $\nabla_{(r, \eta)} \mathcal{A}(\theta, r, \eta ; \varepsilon)=0$ in $\mathcal{V} \times \Lambda_{\varepsilon_{\vartheta}}$, where $\mathcal{V}$ is a neighborhood of $\vartheta$. Note that we have a local solution around each point of $\vartheta \in S^{1}$. Using the compactness of $S^{1}$, and the uniqueness of the solutions $r_{\vartheta}(\theta ; \varepsilon)$ and $\eta_{\vartheta}(\theta ; \varepsilon)$ for $\theta \in \mathcal{V}$ and $\varepsilon<\varepsilon_{\vartheta}$, we can construct functions

$$
r(\theta ; \varepsilon): S^{1} \times \Lambda_{\varepsilon} \rightarrow \mathbb{R}^{+}, \quad \eta(\theta ; \varepsilon): S^{1} \times \Lambda_{\varepsilon} \rightarrow X,
$$

that solve $\nabla_{(r, \eta)} \mathcal{A}(\theta, r, \eta ; \varepsilon)=0$ for $\varepsilon<\varepsilon_{0}=\min _{\vartheta \in S^{1}} \varepsilon_{\vartheta}$. Therefore, critical solutions of $\mathcal{A}$ are critical solutions of $\Psi$.

\section{Comet and moon solutions}

In Proposition 7 we assume that $\alpha \neq 2$, so we analyze two cases separately.

\subsection{Main theorems for $\alpha \geq 1(\alpha \neq 2)$}

Theorem 10 Assume that $\alpha \geq 1(\alpha \neq 2)$ and $\nabla \mathcal{H}=\mathcal{O}_{H_{2 \pi}^{1}}\left(\varepsilon^{2}\right)$, then there is a $\varepsilon_{0}$ such that for $\varepsilon<\varepsilon_{0}$, the functional $\mathcal{A}(x ; \varepsilon)=\mathcal{A}_{0}+\mathcal{H}$ has at least two critical solutions of the form

$$
x(\tau)=e^{J \theta_{j}} x_{0}+\mathcal{O}_{H_{2 \pi}^{1}}\left(\varepsilon^{2}\right)
$$

where $x_{0}=(1,0)$ and $\mathcal{O}_{H_{2 \pi}^{1}}\left(\varepsilon^{2}\right)$ is a $2 \pi$-periodic function of order $\varepsilon^{2}$.

Proof. Since $\nabla \mathcal{H}=\mathcal{O}_{H_{2 \pi}^{1}}\left(\varepsilon^{2}\right)$, by standard estimates in Lyapunov-Schmidt reduction (for instance [10]) we have that $r(\theta ; \varepsilon)=\mathcal{O}\left(\varepsilon^{2}\right)$ and $\eta(\theta ; \varepsilon)=\mathcal{O}_{H_{2 \pi}^{1}}\left(\varepsilon^{2}\right)$ in Proposition 9. By the compactness of $S^{1}$, we conclude that $\Psi$ has at least 2 critical points, one maximum $\theta_{1}$ and one minimum $\theta_{2}$. Since $r(\theta ; \varepsilon)=\mathcal{O}\left(\varepsilon^{2}\right)$ and $\eta(\theta ; \varepsilon)=\mathcal{O}_{H_{2 \pi}^{1}}\left(\varepsilon^{2}\right)$, then $\mathcal{A}(x)$ has at least two critical solutions of the form $x(\tau)=e^{J \theta_{j}} x_{0}+\mathcal{O}_{H_{2 \pi}^{1}}\left(\varepsilon^{2}\right)$.

In the case $\alpha \neq 2$, we obtain the existence of comet solutions as an immediate consequence of Theorem 10 .

Theorem 11 Assume that $\alpha \geq 1(\alpha \neq 2), \sum_{j=1}^{n} m_{j}=1, \omega=\mathfrak{p} / \mathfrak{q}$ and $\nu=1 / \mathfrak{q}$. For each integer $\mathfrak{p}$ there is an integer $\mathfrak{q}_{0}$ such that for each integer $\mathfrak{q}>\mathfrak{q}_{0}$, the restricted $(n+1)$-body problem has at least two $2 \pi \mathfrak{q}$-periodic solutions of the form

$$
q(t)=\varepsilon^{-1} e^{J\left(\theta_{j}+\mathfrak{p} t / \mathfrak{q}\right)} x_{0}+\mathcal{O}(\varepsilon), \quad \varepsilon=(\mathfrak{p} / \mathfrak{q})^{2 /(\alpha+1)} .
$$

Proof. For $\mathfrak{p}$ fixed, there is a $\mathfrak{q}_{0} \in \mathbb{Z}$ such that $\varepsilon=(\mathfrak{p} / \mathfrak{q})^{2 /(\alpha+1)}<\varepsilon_{0}$ if $\mathfrak{q}>\mathfrak{q}_{0}$. From the changes of variables (4) and Theorem 10, after the rescaling $t=\tau / \nu$, for $\varepsilon<\varepsilon_{0}$ the restricted $(n+1)$-body problem has at least two comet solutions of the form

$$
q(t)=\varepsilon^{-1} e^{J \mathfrak{p} t / \mathfrak{q}} x(\tau)=\varepsilon^{-1} e^{J \mathfrak{p} t / \mathfrak{q}}\left(e^{J \theta_{j}} x_{0}+\mathcal{O}\left(\varepsilon^{2}\right)\right),
$$

where the functions $e^{J \mathfrak{p} t / \mathfrak{q}}$ and $\mathcal{O}\left(\varepsilon^{2}\right)$ are $2 \pi \mathfrak{q}$-periodic. 
The comet solution $q(t)$ is $2 \pi \mathfrak{q}$-periodic, then in one period, the $n$ primary bodies travel their $2 \pi$-periodic orbit $\mathfrak{q}$ times, while the comet winds around the origin $\mathfrak{p}$ times.

In the case of moon solutions we only need to assume that $\omega=\mathfrak{p} \nu+1$ and $\omega^{2}=\varepsilon^{-(\alpha+1) / 2}$, i.e. the frequency $\omega$ can take a continuum of values and the solutions that we obtain can be quasiperiodic,

Theorem 12 Assume that $\alpha \geq 1(\alpha \neq 2), q_{j}(t)=e^{J t} a_{j}$ with 10$)$, and $\nu=(\omega-1) / \mathfrak{p}$. From the changes of variables (7) and Theorem 10, for $\varepsilon<\varepsilon_{0}$ we obtain the existence of at least two moon solutions $q(t)$ of the form

$$
q(t)=q_{1}(t)+\varepsilon e^{J\left(\theta_{j}+\omega t\right)} x_{0}+\mathcal{O}\left(\varepsilon^{3}\right), \quad \varepsilon=\omega^{-2 /(\alpha+1)},
$$

where $\mathcal{O}\left(\varepsilon^{3}\right)$ is a (periodic) quasiperiodic function of order $\varepsilon^{3}$.

Actually, the moon solutions are periodic if $\omega=\mathfrak{r} / \mathfrak{q}$ is rational. For the sake of simplicity we consider only the case that $\mathfrak{p}=1$. In those cases we have the following,

Theorem 13 Assume that $\alpha \geq 1(\alpha \neq 2), q_{j}(t)=e^{J t} a_{j}$ with 10$), \omega=\mathfrak{r} / \mathfrak{q}$ and $\nu=\mathfrak{r} / \mathfrak{q}-1$. For each integer $\mathfrak{q}$ there is an integer $\mathfrak{r}_{0}$ such that for each integer $\mathfrak{r}>\mathfrak{r}_{0}$, the restricted $(n+1)$-body problem has at least two $2 \pi \mathfrak{q}$-periodic solutions of the form

$$
q(t)=q_{1}(t)+\varepsilon e^{J\left(\theta_{j}+\mathfrak{r} t / \mathfrak{q}\right)} x_{0}+\mathcal{O}\left(\varepsilon^{3}\right), \quad \varepsilon=(\mathfrak{r} / \mathfrak{q})^{-2 /(\alpha+1)} .
$$

Proof. For $\mathfrak{q}$ fixed, there is a $\mathfrak{r}_{0} \in \mathbb{Z}$ such that $\varepsilon=(\mathfrak{r} / \mathfrak{q})^{-2 /(\alpha+1)}<\varepsilon_{0}$ if $\mathfrak{r}>\mathfrak{r}_{0}$. Thus after the rescaling $t=\tau / \nu$, we obtain solutions

$$
q(t)=q_{1}(t)+\varepsilon e^{J \mathfrak{r} t / \mathfrak{q}}\left(e^{J \theta_{j}} x_{0}+\mathcal{O}\left(\varepsilon^{2}\right)\right),
$$

where the function $\mathcal{O}\left(\varepsilon^{2}\right)$ is $2 \pi / \nu$-periodic in $t$. Since $e^{J \omega t}$ and $\mathcal{O}\left(\varepsilon^{2}\right)$ are $2 \pi \mathfrak{q}$-periodic, then the product $e^{J \omega t} \mathcal{O}\left(\varepsilon^{2}\right)$ is $2 \pi \mathfrak{q}$-periodic.

In the period $2 \pi \mathfrak{r}$ the moon follows a small-amplitude circular orbit of the Kepler problem around the first primary body, where the moon winds around this primary body $\mathfrak{r}$ times, while the primary bodies travel their $2 \pi$-periodic orbit $\mathfrak{q}$ times.

\subsection{Main theorems for $\alpha=2$}

In the case $\alpha=2$, the matrices $A_{\mathfrak{p}}$ and $A_{-\mathfrak{p}}$ in Proposition 7 are not invertible. To avoid this problem we define the action of the group $\mathbb{Z}_{m \mathfrak{p}}$ generated by $\zeta$,

$$
\zeta x(t)=x(t-2 \pi / m \mathfrak{p}), \quad m \geq 2 .
$$

The action functional $\mathcal{A}_{0}$ is $\mathbb{Z}_{m \mathfrak{p}}$-invariant because it does not depend on time explicitly. Therefore, the functional $\mathcal{A}=\mathcal{A}_{0}+\mathcal{H}$ is $\mathbb{Z}_{m \mathfrak{p}}$-invariant when $\mathcal{H}$ is $\mathbb{Z}_{m \mathfrak{p}}$-invariant.

Lemma 14 The action functional $\mathcal{H}$ for the comet is $\mathbb{Z}_{m \mathfrak{p}}$-invariant if there is permutation $\sigma \in S_{n}$ of the set $\{1, \ldots, n\}$ such that

$$
m_{j}=m_{\sigma(j)}, \quad q_{j}(t+2 \pi \mathfrak{q} / m \mathfrak{p})=e^{2 \pi J / m} q_{\sigma(j)}(t), \quad j=1, \ldots, n .
$$

In the case of moon, the action $\mathcal{H}$ is $\mathbb{Z}_{m \mathfrak{p}}$-invariant if the permutation $\sigma$ satisfies $\sigma(1)=1$, $a_{1}=0$ and

$$
m_{j}=m_{\sigma(j)}, \quad a_{j}=e^{2 \pi J / m} a_{\sigma(j)}, \quad j=2, \ldots, n .
$$


Proof. In the comet and moon problems, the action functional $\mathcal{H}(x)=\int_{0}^{2 \pi} h(x, \tau) d \tau$ depends on $\tau$ only through the functions $x_{j}(\tau)$. In the comet case

$$
\mathcal{H}(\zeta x)=\int_{0}^{2 \pi} \sum_{j=1}^{n} m_{j}\left[\phi_{\alpha}\left(\left\|x(\tau)-\varepsilon x_{j}(\tau+2 \pi / m)\right\|\right)-\phi_{\alpha}(\|x(\tau)\|)\right] d \tau=\mathcal{H}(x),
$$

if $m_{j}=m_{\sigma(j)}$ and $x_{j}(\tau+2 \pi / m \mathfrak{p})=x_{\sigma(j)}(\tau)$ for a permutation $\sigma$ of the elements $j \in\{1, \ldots, n\}$. Since $x_{j}(\tau)=e^{-J \mathfrak{p} \tau} q_{j}(\mathfrak{q} \tau)$, because $\omega=\mathfrak{p} / \mathfrak{q}$ and $\nu=1 / \mathfrak{q}$, this holds when

$$
e^{-2 \pi J / m} e^{-J \mathfrak{p} \tau} q_{j}(\mathfrak{q} \tau+2 \pi \mathfrak{q} / m \mathfrak{p})=x_{j}(\tau+2 \pi / m)=x_{\sigma(j)}(\tau)=e^{-J \mathfrak{p} \tau} q_{\sigma(j)}(\mathfrak{q} \tau) .
$$

This condition is equivalent to $q_{j}(\mathfrak{q} \tau+2 \pi \mathfrak{q} / m \mathfrak{p})=e^{2 \pi J / m} q_{\sigma(j)}(\mathfrak{q} \tau)$ and to condition (15).

The same result holds in the moon case if there is a permutation $\sigma$ such that $\sigma(1)=1$ and $x_{j}(\tau+2 \pi / m \mathfrak{p})=x_{\sigma(j)}(\tau)$. Since $(\omega-1) / \nu=\mathfrak{p}$, in this case we have $x_{j}(\tau)=e^{-J \mathfrak{p} \tau} a_{j}$. Therefore, we require that

$$
e^{-2 \pi J / m} e^{-J \mathfrak{p} \tau} a_{j}=x_{j}(\tau+2 \pi / m \mathfrak{p})=x_{\sigma(j)}(\tau)=e^{-J \mathfrak{p} \tau} a_{\sigma(j)} .
$$

This condition holds only when $a_{j}=e^{2 \pi J / m} a_{\sigma(j)}$. Since $\sigma(1)=1$, we require that $a_{1}=0$.

Theorem 15 Under the assumptions (15) for the comet solutions, and (16) for the moon solutions, the same results as in Theorems 11 and 13 hold in the gravitational case with $\alpha=2$.

Proof. The subspace of fixed points under the $\mathbb{Z}_{m \mathfrak{p}}$-action is

$$
\left(H_{2 \pi}^{1}\right)^{\mathbb{Z}_{m \mathfrak{p}}}=\left\{x: \sum_{l \in \mathbb{Z}} x_{j} e^{i l(t+\pi)}=\sum_{l \in \mathbb{Z}} x_{j} e^{i l t}\right\}=\left\{x: x_{l}=0 \quad \text { for } \quad l=0, \pm m \mathfrak{p}, \pm 2 m \mathfrak{p}, \ldots\right\}
$$

By the Palais Criticality Principle, a critical point of $\mathcal{A}:\left(H_{2 \pi}^{1}\right)^{\mathbb{Z}_{m \mathfrak{p}}} \rightarrow \mathbb{R}$ is a critical point of the action $\mathcal{A}: H_{2 \pi}^{1} \rightarrow \mathbb{R}$. Then, we can apply the previous method in the fixed point space $\left(H_{2 \pi}^{1}\right)^{\mathbb{Z}_{m \mathfrak{p}}}$. The result follows from the fact that the Hessian $\nabla^{2} \mathcal{A}_{0}\left(e^{J \theta} a_{0}\right)$ is invertible in $\left(H_{2 \pi}^{1}\right)^{\mathbb{Z}_{m \mathfrak{p}}}$ if $m \geq 2$.

Remark 16 For example, the choreographies in [7] have $n$ bodies forming m-polygons at any time. In these solutions there is a permutation $\sigma$ with $\sigma^{m}=(1)$ such that

$$
q_{j}(t)=e^{2 \pi J / m} q_{\sigma(j)}(t) .
$$

The condition (15) is satisfied by the choreographies with the condition (17) for $\mathfrak{q}=\operatorname{lm} \mathfrak{p}$ with $l \in \mathbb{N}$. A particular example of such choreographies is the super-eight choreography (see Figure 2) that satisfies the symmetry

$$
q_{j}(t)=e^{J \pi} q_{\sigma(j)}(t)=-q_{\sigma(j)}(t),
$$

where $\sigma=(13)(24)$ is the permutation of order $m=2$.

Remark 17 An example of a central configuration that satisfies condition (16) is the Maxwell configuration, which consists of a central mass $a_{1}=0$ and $(n-1)$-equal masses at the vertices of a polygon $a_{j}=e^{2 \pi J / m} x_{0}$ with $m=n-1$. The Maxwell configuration satisfies condition (16) for the permutation $\sigma=(23 \ldots n-1)$ of order $m$. Similar configurations that satisfy the condition (16) consist of a central mass with an arrangement of nested polygons in [14] . 


\section{$5 \quad$ Numerical study of periodic orbits}

The variational method in the previous section has some limitations due to assumptions imposed on the solution of the primary bodies. This section is dedicated to exploring numerically, with the reversibility technics in [24], the existence of comet and moon solutions for four primaries following the super-eight choreography. The super-eight choreography is a periodic solution of the 4-body problem where four primaries with unitary mass follow periodically the same path. We assume that the origin of the coordinate system is located at the center of mass.

\subsection{Super-eight choreography of the 4-body problem}

The super-eight choreography has two special kinds of configurations that correspond to fixed points of transformations for positions and velocities. These special transformations are called reversing symmetries [18], and we refer to its fixed points as reversible configurations. We use this technique to obtain periodic orbits in the restricted 5-body problem.

During the temporal evolution of the super-eight choreography two kind of reversible configurations appear at $\bar{T}:=\pi / 4$-units of time, where $T:=2 \pi$ is the period of the orbit. One of these reversible configurations, called isosceles configuration, has the characteristic that the bodies are located at the vertices of two isosceles triangles where one vertex is the center of mass of the system. The velocities of the bodies are related by a reflection along the basis of the corresponding isosceles triangle. The positions $q_{j}$ and velocities $v_{j}=\dot{q}_{j}$ of the isosceles configurations, for time $t=0$, is given by

$$
\begin{gathered}
q_{1}=(0.939977120285667,-0.327721385645527)^{T}, \\
q_{2}=K q_{1}, \quad q_{3}=-q_{1}, \quad q_{4}=-q_{2}, \\
v_{1}=(1.122200245052303,-0.117392625737923)^{T}, \\
v_{2}=-K v_{1}, \quad v_{3}=-v_{1}, \quad v_{4}=-v_{2},
\end{gathered}
$$

where

$$
K=\left(\begin{array}{cc}
1 & 0 \\
0 & -1
\end{array}\right) .
$$

At time $t=\bar{T}$ the four bodies pass by other kind of reversible configuration. The geometric property of this configuration is that the positions and velocities of the bodies are orthogonal; we called it orthogonal configuration. The positions and velocities of the four bodies at $t=\bar{T}$ are

$$
\begin{gathered}
q_{1}=(1.382856843618412,0)^{T}, \quad q_{2}=(0,0.157029922281204)^{T}, \\
q_{3}=-q_{1}, \quad q_{4}=-q_{2}, \\
v_{1}=(0,0.584872630814899)^{T}, v_{2}=(1.871935245878693,0)^{T}, \\
v_{4}=-v_{2}, \quad v_{3}=-v_{1} .
\end{gathered}
$$

The isosceles and orthogonal reversible configurations of the Gerver's super eight are shown in Fig. 2. We remark that these configurations are fixed points of reversing symmetries in the 4-body problem with equal masses [24]. 


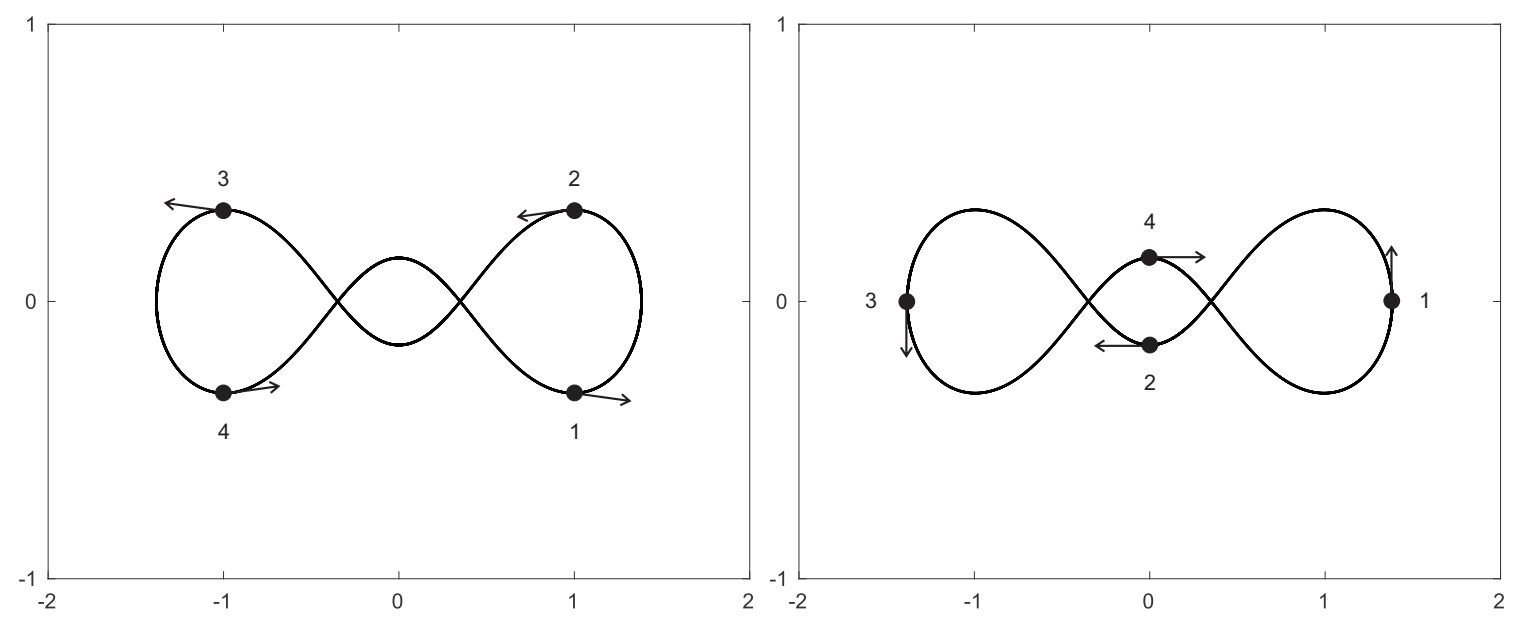

Figure 2. Gerver's super eight choreography. At left an isosceles reversible configuration, at time $t=0$. At right an orthogonal reversible configuration, at time $t=\bar{T}$.

\subsection{The restricted 5-body problem for the super-eight choreography}

Consider a system of five particles, four of them with unitary mass $m_{i}=1, i=1,2,3,4$, following the periodic super-eight choreography, and the fifth $i=5$ as a test particle moving under the force exerted by the other four. The equations of motions of the five particles are given by the expressions

$$
\ddot{q}_{i}(t)=-\sum_{j=1, j \neq i}^{5} m_{j} \frac{q_{i}(t)-q_{j}(t)}{\left\|q_{i}(t)-q_{j}(t)\right\|^{3}}, \quad i=1, \cdots, 5 .
$$

We assume that the origin of the coordinate system coincides with the center of mass of the choreographic bodies. We restrict (21) in such a way the bodies with unitary mass always follow the super-eight choreography. So, for the bodies with unitary mass we choose as initial conditions (18) or (20). In consequence, only the initial conditions of the fifth particle are unknown. Our aim is to determine initial conditions of the fifth particle that lead to periodic orbits of comet or moon type. We will consider reversible configurations of the restricted 5-body problem that are consistent with the Gerver's super-eight choreography as we explain in the following sections.

\subsection{Reversing symmetries of the restricted five-body problem}

In order to introduce the results related with reversing symmetries, we need to rewrite the equations of motion (21). For this aim, we introduce the state vector of the five bodies

$$
u(t)=\left(q_{1}(t), \cdots, q_{5}(t), v_{1}(t), \cdots, v_{5}(t)\right) .
$$

The equations of motion can be written as

$$
\dot{u}(t)=\left(v_{1}(t), \cdots, v_{5}(t), a_{1}(t), \cdots, a_{5}(t)\right),
$$

where

$$
a_{i}(t)=-\sum_{j=1, j \neq i}^{5} m_{j} \frac{q_{i}(t)-q_{j}(t)}{\left\|q_{i}(t)-q_{j}(t)\right\|^{3}}, \quad i=1, \cdots, 5 .
$$


With this, the equation (21) becomes

$$
\frac{d u(t)}{d t}=F(u(t)), \quad\left(F_{2 i-1}, F_{2 i}\right)=q_{i}(t), \quad\left(F_{8+2 i-1}, F_{8+2 i}\right)=a_{i}(t), \quad i=1, \cdots, 5 .
$$

We notice that system (24) is not defined at collisions. That is, defining

$$
\Delta_{i j}=\left\{\left(q_{1}, \cdots, q_{5}\right) \in \mathbb{R}^{10} \quad \mid \quad q_{i}=q_{j}\right\}, \quad \Delta=\bigcup_{i<j} \Delta_{i j}
$$

we have that the system (24) is defined only for $u \in \Omega=\mathbb{R}^{10} \backslash \Delta \times \mathbb{R}^{10}$.

Definition 18 Given the ordinary differential equation (24), we say that an involution $R$ : $\Omega \rightarrow \Omega$ is a reversing symmetry if

$$
\frac{d}{d t} R(u(t))=-F(R(u(t)))
$$

holds. The set of fixed points of $R$ is denoted as $\mathbf{F i x}(R)$.

The reversing symmetries are useful for determining symmetric periodic orbits. The Muñoz-Almaraz's Theorem [25] gives the relation between the reversing symmetries and symmetric periodic orbits. We state this theorem applied to our restricted 5-body problem.

Theorem 19 Let $u(t)=\left(q_{1}(t), \cdots, q_{5}(t), v_{1}(t), \cdots, v_{5}(t)\right)$ be a well-defined solution of the 5 -body problem in the time interval $\left[0, T_{0}\right]$. Suppose that the solution $u(t)$ passes through fixed points of reversing symmetries $R$ and $\widehat{R}$ at times $t=0$ and $t=T_{0}$, respectively. Then the solution is defined for all $t \in \mathbb{R}$, and we have for all $m \in \mathbb{Z}$ that

$$
\begin{gathered}
u(-t)=R u(t), \\
u(t)=\widehat{R} u\left(2 T_{0}-t\right), \\
u\left(2 m T_{0}+t\right)=(\widehat{R} R)^{m} u(t) .
\end{gathered}
$$

In addition, if there exists some $M \in \mathbb{N}$ such that $(\widehat{R} R)^{M}=i d$ then $u(t)$ is periodic, with period $T=2 M T_{0}$.

In order to apply Theorem 19 to the restricted 5-body problem, we need to consider fixed points of reversing symmetries of that problem. The super-eight choreography is a solution of the 4-body problem that passes through fixed points of specific reversing symmetries of the 4-body problem. In our case, we consider reversing symmetries of the 5-body problem that are consistent with the super-eight choreography. We remark that these reversing symmetries of the 5-body problem also hold for our restricted 5-body problem. There are several reversing symmetries that allow to obtain periodic orbits of comet and moon type. We only consider the reversing symmetries

$$
\begin{aligned}
& q_{1} \rightarrow K q_{2}, \quad v_{1} \rightarrow-K v_{2}, \quad q_{1} \rightarrow K q_{2}, \quad v_{1} \rightarrow-K v_{2}, \\
& q_{2} \rightarrow K q_{1}, \quad v_{2} \rightarrow-K v_{1}, \quad q_{2} \rightarrow K q_{1}, \quad v_{2} \rightarrow-K v_{1}, \\
& \Phi_{1 x}: q_{3} \rightarrow K q_{4}, \quad v_{3} \rightarrow-K v_{4}, \quad \Phi_{1 y}: q_{3} \rightarrow K q_{4}, \quad v_{3} \rightarrow-K v_{4}, \\
& q_{4} \rightarrow K q_{3}, \quad v_{4} \rightarrow-K v_{3}, \quad q_{4} \rightarrow K q_{3}, \quad v_{4} \rightarrow-K v_{3}, \\
& q_{5} \rightarrow K q_{5}, \quad v_{5} \rightarrow-K v_{5}, \quad q_{5} \rightarrow-K q_{5}, \quad v_{5} \rightarrow K v_{5},
\end{aligned}
$$




$$
\begin{aligned}
& q_{1} \rightarrow K q_{1}, \quad v_{1} \rightarrow-K v_{1}, \quad q_{1} \rightarrow K q_{1}, \quad v_{1} \rightarrow-K v_{1}, \\
& q_{2} \rightarrow-K q_{2}, \quad v_{2} \rightarrow K v_{2}, \quad q_{2} \rightarrow-K q_{2}, \quad v_{2} \rightarrow K v_{2}, \\
& \Psi_{1 x}: q_{3} \rightarrow K q_{3}, \quad v_{3} \rightarrow-K v_{3}, \quad \Psi_{1 y}: q_{3} \rightarrow K q_{3}, \quad v_{3} \rightarrow-K v_{3}, \\
& q_{4} \rightarrow-K q_{4}, \quad v_{4} \rightarrow K v_{4}, \quad q_{4} \rightarrow-K q_{4}, \quad v_{4} \rightarrow K v_{4}, \\
& q_{5} \rightarrow K q_{5}, \quad v_{5} \rightarrow-K v_{5}, \quad q_{5} \rightarrow-K q_{5}, \quad v_{5} \rightarrow K v_{5},
\end{aligned}
$$

where $K$ is the matrix defined in $(19)$. The set $\operatorname{Fix}\left(\Phi_{1 x}\right)$ is given by the points $(22)$, where $q_{j}=\left(q_{j x}, q_{j y}\right), v_{j}=\left(v_{j x}, v_{j y}\right), j=1, \cdots, 5$ satisfy

$$
\begin{aligned}
& q_{2 x}=q_{1 x}, \quad q_{2 y}=-q_{1 y}, \quad q_{3 x}=-q_{1 x}, \quad q_{3 y}=-q_{1 y}, \quad q_{4 x}=-q_{2 x}, \quad q_{4 y}=-q_{2 y}, \quad q_{5 y}=0 \\
& v_{2 x}=-v_{1 x}, \quad v_{2 y}=v_{1 y}, \quad \dot{q}_{3 x}=-v_{1 x}, \quad v_{3 y}=-v_{1 y}, \quad v_{4 x}=-v_{2 x}, \quad v_{4 y}=-v_{2 y}, \quad v_{5 x}=0 .
\end{aligned}
$$

On the other hand, for the set $\operatorname{Fix}\left(\Psi_{1 x}\right)$ we have

$$
\begin{aligned}
& q_{1 y}=0, \quad q_{2 x}=0, \quad q_{3 x}=-q_{1 x}, \quad q_{3 y}=-q_{1 y}, \quad q_{4 x}=-q_{2 x}, \quad q_{4 y}=-q_{2 y}, \quad q_{5 y}=0 \\
& v_{1 x}=0, \quad v_{2 y}=0, \quad v_{3 x}=-v_{1 x}, \quad v_{3 y}=-v_{1 y}, \quad v_{4 x}=-v_{2 x}, \quad v_{4 y}=-v_{2 y}, \quad v_{5 x}=0 .
\end{aligned}
$$

The sets $\operatorname{Fix}\left(\Phi_{1 y}\right)$ and $\operatorname{Fix}\left(\Psi_{1 y}\right)$ are obtained from (25) and (26), respectively, by replacing the conditions for the fifth particle by $q_{5 x}=0, v_{5 y}=0$. We say that $\operatorname{Fix}\left(\Phi_{1 x}\right)$ and $\operatorname{Fix}\left(\Phi_{1 y}\right)$ are isosceles reversible configurations, whereas $\operatorname{Fix}\left(\Psi_{1 x}\right)$ and $\operatorname{Fix}\left(\Psi_{1 y}\right)$ orthogonal reversible configurations, of the restricted five-body problem. We remark that these reversible configurations hold for both full and restricted 5-body problems. For the restricted 5-body problem, we are interested in those orbits that pass through two fixed points of some reversing symmetries. We will consider specific combinations of reversing symmetries that lead to comet and moon orbits.

\subsection{Comet orbits}

For the numerical computation of comet orbits of the restricted 5-body problem, we consider two different combinations of reversible configurations. First, we deal with the solutions that pass through points within $\operatorname{Fix}\left(\Phi_{1 x}\right)$ at $t=0$, and $\operatorname{Fix}\left(\Phi_{1 y}\right)$ at some time $t=T_{0}$. According to the initial condition of the choreographic bodies (18), the orbit of the four primary bodies is consistent with $\operatorname{Fix}\left(\Phi_{1 y}\right)$ if and only if $T_{0}=4 m \bar{T}, m \in \mathbb{Z}$. Thus, if we have a solution $u(t)$ which meet $u(0) \in \operatorname{Fix}\left(\Phi_{1 x}\right), u\left(T_{0}\right) \in \operatorname{Fix}\left(\Phi_{1 y}\right)$ with $T_{0}=4 m \bar{T}$ for some $m \in \mathbb{N}$, by Theorem 19 and the relation between the reversing symmetries

$$
\left(\Phi_{1 y} \Phi_{1 x}\right)^{2}=i d
$$

we get that $u(t)$ is periodic (in inertial frame) with period $T=4 T_{0}$. For a second combination of reversing symmetries consider $u(t)$ such that $u(0) \in \operatorname{Fix}\left(\Phi_{1 x}\right), u\left(T_{0}\right) \in \operatorname{Fix}\left(\Phi_{2 y}\right)$ with $T_{0}=(4 m+2) \bar{T}$ for some $m \in \mathbb{N}$. In this case the orbit is periodic since $\left(\Phi_{2 y} \Phi_{1 x}\right)^{2}=i d$, with period $T=4 T_{0}$.

In order to compute numerically the initial conditions of $u(t)$ for the first combination (for the second one we could follow a similar approach), we need to find a time $T_{0}=4 m \bar{T}$ with $m \in \mathbb{N}$, and initial conditions $q_{5}=(\alpha, 0), v_{5}=(0, \beta), \alpha, \beta \in \mathbb{R}$, in such a way the equalities

$$
\begin{aligned}
& \phi_{0}(\alpha, 0,0, \beta)=(\alpha, 0,0, \beta), \\
& \phi_{T_{0}}(\alpha, 0,0, \beta)=(\gamma, 0,0, \delta),
\end{aligned}
$$




\begin{tabular}{|c|c|c|}
\hline $\bar{T}$ & $\alpha$ & $\beta$ \\
\hline $2 \pi$ & 4.116104103490420 & 1.044999754887220 \\
\hline $5 \pi / 2$ & 4.742060123223827 & 0.958945634262276 \\
\hline $3 \pi$ & 5.330615961036938 & 0.896037359621114 \\
\hline $7 \pi / 2$ & 5.889293694917488 & 0.847128753375993 \\
\hline $4 \pi$ & 6.423300718815878 & 0.807515201172657 \\
\hline$\pi / 2$ & 1.469992697921058 & 3.966907060848269 \\
\hline
\end{tabular}

Table 1. Positions and velocities $q_{5}=(\alpha, 0), v_{5}=(0, \beta)$ of the fifth particle that give rise to periodic orbits in the restricted 5-body problem. The first five rows and 18 define initial conditions of comet orbits. On the other hand, the sixth row and (20) define initial conditions of moon orbits.

hold. Here $\phi$ is the flow associated to the equations of motions (restricted problem), and $\gamma, \delta$ are arbitrary real numbers. The first equation (27) establish the condition $u(0) \in \operatorname{Fix}\left(\Phi_{1 x}\right)$, whereas the second one $u\left(T_{0}\right) \in \operatorname{Fix}\left(\Phi_{1 y}\right)$. In this case both $q_{5}=(\alpha, 0), v_{5}=(0, \beta)$ and (18) define an initial condition of a periodic orbit of comet type in the restricted 5-body problem. For details about the numerical computation of these orbits the reader is referred to the restricted 4-body problem in [19].

The main property of the comet orbits is that the test particle is located far away from the primaries. In that case, the primaries exert a force approximately equivalent to that of a body with mass $m=4$ over the test particle. In Figure 1 we have shown several orbits of this type and in Table 1 the corresponding initial conditions.

\subsection{Moon orbits}

The moon orbits are the opposite of the comet orbits, they are characterized by the property that the test particle moves around some of the primaries, acting as satellite. In this case these bodies conform approximately a binary system. We assume that the primary involved with the test particle is the body with index $i=1$.

In order to compute periodic orbits of moon type we also use the technic of reversing symmetries. In this case the isosceles reversible configuration of the restricted 5-body problem is not consistent with the super-eight choreography and the moon orbits. Nevertheless, the orthogonal reversible configurations are consistent with the super-eight choreography and the moon orbits, which are precisely the fixed points of $\Psi_{1 x}$ and $\Psi_{1 y}$. In order to define a boundary value problem where the initial condition is involved, we make a temporal shift of $\bar{T}$ units in the equations of motion (21). Therefore, by means of introducing the new time $\tau=t-\bar{T}$, the orthogonal reversible configuration which happens at $t=\bar{T}$ is associated to the new time $\tau=0$. Thus, consider a solution $u(\tau)$ in such a way $u(0) \in$ Fix $\left(\Psi_{1 x}\right)$ and $u\left(T_{0}\right) \in$ Fix $\left(\Psi_{1 y}\right)$, which can happen only if $T_{0}=4 m \bar{T}$ for some $m \in \mathbb{N}$. According to Theorem 19, and the relation

$$
\left(\Psi_{1 y} \Psi_{1 x}\right)^{2}=i d,
$$

the solution $u(\tau)$ is periodic (in inertial frame), with period $T=4 T_{0}$.

In order to compute the initial conditions of periodic orbits, we proceed as we did for the comet case (see (27)). We have to find a time $T_{0}=4 m \bar{T}$ with $m \in \mathbb{N}$, and values $\alpha, \beta \in \mathbb{R}$, in 
such a way that the equalities

$$
\begin{aligned}
& \phi_{0}(\alpha, 0,0, \beta)=(\alpha, 0,0, \beta), \\
& \phi_{T_{0}}(\alpha, 0,0, \beta)=(0, \gamma, \delta, 0),
\end{aligned}
$$

are fulfilled, where $\gamma, \delta$ are arbitrary real numbers. The first equation $(28)$ implies that $u(0) \in$ $\operatorname{Fix}\left(\Psi_{1 x}\right)$, whereas the other one that $u\left(T_{0}\right) \in \operatorname{Fix}\left(\Psi_{1 y}\right)$. The initial condition of the restricted 5 -body problem (with shifted time) is given by $q_{5}=(\alpha, 0), v_{5}=(0, \beta)$ and (20). In Figure 1 we have shown a periodic and symmetric moon orbit of the restricted 5-body problem; in Table 1 we give the corresponding initial condition.

\section{References}

[1] Arribas, M., Elipe, A.: Bifurcations and equilibria in the extended $n$-body ring problem. Mech Res Comm 31(1):1-8, 2004.

[2] Bang, D., Elmabsout, B.: Restricted $n+1$-body problem: existence and stability of relative equilibria. Celestial Mech Dynam Astronom 89(4):305-318, 2004.

[3] Beltritti, G., Mazzone, F., Oviedo, M.: A generalized Sitnikov Problem. arXiv:1710.02667, 2017.

[4] Bengochea, A., Galán, J., Pérez-Chavela, E.: Doubly-symmetric horseshoe orbits in the general planar three-body problem. Astrophys Space Sci. 348 (2013) 403-415.

[5] Bengochea, A., Galán, J., Pérez-Chavela, E.: Exchange orbits in the planar 1+4 body problem. Physica D 301-302 (2015) 21-35.

[6] Broucke, R.: Stability of periodic orbits in the elliptic, restricted three-body problem. AIAA Journal. (1969) 1003-1009 (doi:10.2514/3.5267).

[7] Calleja, R., Doedel, E., García-Azpeitia, C.: Symmetries and choreographies in families bifurcating from the polygonal relative equilibrium of the n-body problem. Celestial Mech. Dyn. Astr. (2018) 130: 48. (doi:10.1007/s10569-018-9841-9).

[8] Chenciner, A., Montgomery, R.: A remarkable periodic solution of the three-body problem in the case of equal masses. Ann. Math 152 (2000) 881-901.

[9] Doedel, E., Romanov, V., Paffenroth, R., Keller, H., Dichmann, D., Galán-Vioque, J., Vanderbauwhede, A.: Elemental periodic orbits associated with the libration points in the circular restricted 3-body problem. Bifurcation and Chaos 17(8):2625-2677, 2007.

[10] Fontaine, M., García-Azpeitia, C.: Braids of then-body problem by cabling central configurations.

[11] Fontaine, M., Montaldi, J.: Persistence of stationary motion under explicit symmetry breaking perturbation.

[12] Galán, J., Muñoz-Almaraz, F. J., Freire, E., Doedel, E.: Stability and Bifurcations of the Figure-8 Solution of the Three-Body Problem. Phys. Rev. Lett. 88 (2002) 241101. 
[13] García-Azpeitia, C., Ize, J.: Global bifurcation of planar and spatial periodic solutions in the restricted n-body problem. Celestial Mech. Dyn. Astr. 110 (2011) 217-227 (doi:10.1007/s10569-011-9354-2).

[14] García-Azpeitia, C., Ize, J.: Global bifurcation of polygonal relative equilibria for masses, vortices and dNLS oscillators. J. Differential Equations 251 (2011) 3202-3227 (doi:10.1016/j.jde.2011.06.021).

[15] Gómez, G., Simó, C., Llibre, J., Martinez, R.: Dynamics and mission design near libration points. Vol. 1-4. World Scientific Monograph Series in Mathematics, World Scientific, 2000 .

[16] Kalvouridis, T.J.: Particle motions in Maxwell's ring dynamical systems. Celest Mech Dyn Astron 102(1-3):191-206, 2008.

[17] Kapela, T., Zgliczyńsk, P.: The existence of simple choreographies for the $N-$ body problem - a computer-assisted proof. Monogr. Nonlinearity 16 (2003) 1899-1918 (doi:10.1088/0951-7715/16/6/302).

[18] Lamb, J.S.W., Roberts, J.A.G.: Time-reversal symmetry in dynamical systems: a survey. Physica D 112 (1998) 1-39 (doi:10.1016/S0167-2789(97)00199-1).

[19] Lara, R., Bengochea, A.: A restricted four-body problem for the eight figure choreography. Submitted to Regul. Chaotic Dyn.

[20] Llibre, J., Stoica, C.: Comet and Hill-type periodic orbits in restricted $(N+1)$-body problems. Journal of Differential Equations 250: 1747-1766, 2011.

[21] Maciejewski, A., Rybicki, S.: Global bifurcations of periodic solutions of the restricted three body problem. Celestial Mechanical and Dynamical Astronomy 88:293-324, 2004.

[22] Meyer, K., Hall, G.: An Introduction to Hamiltonian Dynamical Systems and the Nbody problem. Springer-Verlag, 1991.

[23] Moser, J., Zehnder, E.: Notes on Dynamical Systems. Courant Lecture Notes, 2006.

[24] Muñoz-Almaraz, F.J., Freire, E., Galán, J., Vanderbauwhede, A.: Continuation of Gerver's supereight choreography. Monogr. Real Acad. Ci. Exact. Fís.-Quím. Nat. Zaragoza 30 (2006) 95-105.

[25] Muñoz-Almaraz, F.J., Freire, E., Galán J., Vanderbauwhede, A. : Continuation of normal doubly symmetric orbits in conservative reversible systems. Cel. Mech. Dynam. Astronom. 97 (2007) 17-47 (doi:10.1007/s10569-006-9048-3).

[26] Shibayama, M.: Variational Proof of the Existence of the Super-Eight Orbit in the Four-Body Problem. Arch. Rational Mech. Anal. 1 (2014) 77-98 (doi:10.1007/s00205014-0753-x).

[27] Sicardy, B.: Stability of the triangular Lagrange points beyong Gaschau's value. Celest Mech Dyn Astron 107: 145-155, 2010. 
[28] Szebehely, V., Giacaglia, G. E. O.: On the elliptic restricted problem of three bodies. AJ. 69 (1964) 230-235. 